View Point

\title{
Key to Normalcy - Recognising the Extent of COVID-19 Pandemic
}

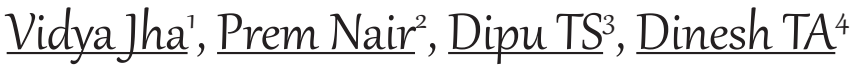

\begin{abstract}
${ }^{1}$ Assistant Professor, Asst. Medical Administrator, Amrita Institute of Medical Sciences and Research Center, Amrita Vishwa Vidyapeetham University, Cochin, Kerala, India.

${ }^{2}$ Professor, Department of Hospital Administration, Amrita Institute of Medical Sciences and Research Center, Amrita Vishwa Vidyapeetham University, Cochin, Kerala, India.

${ }^{3}$ Associate Professor, Department of Infectious Diseases, Amrita Institute of Medical Sciences and Research Center, Amrita Vishwa Vidyapeetham University, Cochin, Kerala, India.

${ }^{4}$ Professor \& HOD, Master of Hospital Administration, Amrita Institute of Medical Sciences and Research Center, Amrita Vishwa Vidyapeetham University, Cochin, Kerala, India.
\end{abstract}

DOI: https://doi.org/10.24321/2455.7048.202006

\section{I $\quad \mathbf{N} \quad \mathbf{F} \quad \mathbf{O}$}

\author{
Corresponding Author: \\ Dr. Vidya Jha, Asst. Medical Administrator, Amrita \\ Institute of Medical Sciences and Research \\ Center, Amrita Vishwa Vidyapeetham University, \\ Cochin, Kerala, India. \\ E-mail Id: \\ vidyajha29@gmail.com \\ Orcid Id: \\ https://orcid.org/0000-0001-7524-3566 \\ How to cite this article: \\ Jha V, Nair P, Dipu TS, Dinesh TA. Key to Normalcy \\ - Recognising the Extent of COVID-19 Pandemic. \\ Epidem Int 2020; 5(1): 29-31.
}

Date of Submission: 2020-04-03

Date of Acceptance: 2020-04-08
The current COVID-19 outbreak containment measures till date were primarily based on early detection, isolation and social distancing. This has been aided by the testing of nasopharyngeal swab of the suspect's using RT-PCR. The above measures are aimed to "flatten the curve" so that the healthcare system will not be overwhelmed. As the community spread widens and the cases are on the rise $(120 \%$ increase in cases over the last 5 days in India), relying solely on the RT-PCR based testing of viral genome detection would be grossly inadequate as a containment strategy (as of $6^{\text {th }}$ April the number of tests done in India is 102 tests per million population while compared to USA's and Italy's 5355 and 11436 tests/million respectively). Active replication of the virus is needed to get a positive test. In a society of billion people only a subset of the people could be anticipated to have active viral shedding. A wider base which includes silent carriers and those who recovered from the illness have to be detected and it would need Antibody based testing. As the antibody response stays for a longer period, the practical applications of these antibody-based tests range from clinical diagnostics, therapeutics to understanding the epidemiology and making strategic plans.

This test is also known as "serological" test or "immunity" test and it has been rolled out in India too. This test will help the health officials and the decision makers in answering some basic questions on COVID-19 and the causative virus SARS-CoV-2. For a better epidemiological understanding we need to find out correct answers for questions like how many people have been infected with this virus? Why do some people respond mildly while others become severely ill? How deadly is SARS-CoV-2? Who may have unknowingly transmitted it? What measures are actually effective in slowing its spread?

The serological test detects whether an individual has developed antibodies against SARS-CoV-2 thereby indicating whether the individual 
had been a carrier at one point of time and now has developed immunity. It will help in identifying people who are immune and the entire extent of the COVID-19 pandemic can be revealed through this test.

The current available diagnostic tests for coronavirus globally are based on looking for genetic sequence of the virus. These tests require nasal or throat swabs to be taken by trained personnel and are then sent to a specialised laboratory for analysis. Such genetic tests detect only the active infections. In contrast, serological/antibody tests detect the antibodies that our bodies produce in order to kill the virus and this is produced even after we have eliminated the virus. One can also identify who had been infected even after their recovery from COVID-19. The test kit requires only a drop of blood, is cheaper and the turnaround time is $\sim 10-15$ minutes. The other advantage is that the kit can be produced on a mass scale cheaply and quickly.

This serological test is less useful in finding an active infection as it can take several days for a person to make these antibodies after COVID. It can yield a false negative result too, i.e it shows that a person does not have coronavirus when they actually do.

Germany is considering using these serological tests to issue "immunity certificates" to people who have survived Covid-19. ${ }^{1}$ Those who show immunity could be given a type of vaccination card that allows them to be exempted from COVID related restrictions on their work and travel.

Towards the end of March 2020, the United Kingdom had also ordered 3.5 million serological tests. ${ }^{2}$

We believe, India with a population of 1.38 billion, this test might turn out to be essential on the way to normalcy as it will also help in detection of individuals who were never diagnosed with COVID-19 either because they were asymptomatic, or had just mild illness or could not get an initial test done due to affordability or availability issues. This will allow government and the decision makers to ease restrictions and lockdown.

Individuals who test positive can be considered to be immune/safe from another infection at least for some time (coronavirus being a novel virus - we have limited knowledge about it). Due to mutation, the virus could render past immunity ineffective. However, researchers have found it to mutate slowly which indicates that protection from past infection may be effective for at least a while.

This will indicate who can be prioritized to return to work especially in health sector where the number of health professionals being exposed to coronavirus and testing positive is increasing globally.

However, one should be critical in choosing the serological test kit companies. Recently the Spanish government withdrew 58,000 Chinese-made coronavirus testing kits from use after it emerged that they had an accurate detection rate of just $30 \%{ }^{3}$

On $2^{\text {nd }}$ April 2020, the Food and Drug Administration (FDA) approved a serological test for COVID-19 with an emergency use authorization in USA. ${ }^{4}$ FDA has also recently allowed doctors to use blood plasma from recovered patients that contains antibodies to treat emergency cases of COVID-19 and these serological tests can identify potential antibody donors. ${ }^{5}$

Coming back to the strategy to combat the COVID-19 pandemic and return to normalcy, the main task in hand is to identify who currently has the virus, thereby making RT-PCR tests the priority. But as India goes deeper into the pandemic, situation will demand serological tests that will become key to normalcy. This combination to identify both active and past infections will serve to be a highly effective strategy. Therefore, it should include serological tests coupled with RT-PCR tests to look for active cases of Covid-19. This will help health officials to identify hot spots and the government can more precisely target their tactics/interventions. Rather than blanket lockdown, sealing of inter-state borders, decision makers can identify which cities, localities and group of people are most at risk and initiate relaxation of quarantines, curfews, lockdowns and other control measures in some areas.

Another important measure that India should be interested in starting is "serosurveys" meaning estimation of spread of virus by testing the blood of a sample of the population. Such retrospective initiatives will help in approximating the full number of cases which can help explain how common asymptomatic COVID-19 may be and also better estimate mortality rate of a virus.

At a community level, if a "serosurvey" indicates higher number of immune people than realized, it could indicate that future episodes of coronavirus cases might be less intense than some forecasts anticipate it to be. Looking ahead in future when a potential vaccine is developed, such a "serosurvey" could also ensure that people who have not contracted coronavirus yet could be prioritised over the immune people. World Health Organisation (WHO) is also urging countries to take up such serosurveys and share the results. This will help us in bringing back normalcy as well as make us better prepared for next epidemic, if it happens.

Duration of lockdown or Social Distancing (SD) is critical in containing the coronavirus. If it is lifted too early, the population may experience a second epidemic peak as susceptible individuals who were protected till now may be exposed. Such a situation has been reported by Bootsma and Ferguson who analysed SD interventions in 16 United 
States cities during the 1918 epidemic. ${ }^{6}$ Knowing the full extent of COVID-19 pandemic with the help of serological tests will guide us in taking better decisions.

Therefore, the authors believe that identification of both active infections (by RT-PCR) and past infections (antibodybased test) along with compliance to social distancing can decelerate the spread of the virus. It will serve to be a highly effective strategy and eventually help gain momentum in re-strengthening Indian economy.

\section{Conflicts of Interest: None}

\section{References}

1. https://www.businessinsider.in/science/news/ germany-could-issue-hundreds-of-thousands-ofpeople-with-coronavirus-immunity-certificates-sothey-can-leave-the-lockdown-early/ articleshow/ 74894558.cms?utm_source= contentofinterest \&utm _ medium=text\&utm_campaign=cppst.

2. https://www.newscientist.com/article/2238834-willa-home-antibody-test-for-covid-19-really-be-a-gamechanger/\#ixzz6leaJzAKQ

3. https://www.theguardian.com/world/2020/mar/27/ coronavirus-test-kits-withdrawn-spain-poor-accuracyrate.

4. https://www.nytimes.com/2020/04/02/health/ coronavirus-antibody-test.html.

5. https://www.fda.gov/vaccines-blood-biologics/ investigational-new-drug-ind-or-device-exemption-ideprocess-cber/investigational-covid-19-convalescentplasma-emergency-inds.

6. Bootsma MC, Ferguson NM (2007). "The effect of public health measures on the 1918 influenza pandemic in U.S. cities". Proc Natl Acad Sci U S A. 104 (18): 75887593. doi:10.1073/pnas.0611071104. PMC 1849868. PMID 17416677. 